\title{
Mobile Agents Integrity Research
}

\author{
Huanmei Guan ${ }^{1,2}$, Huanguo Zhang ${ }^{2,3}$, Ping Chen ${ }^{1}$, and Yajie Zhou ${ }^{1}$ \\ 1 Computer Center, Wuhan University, Wuhan 430072, China \\ hmguan-wh@163.com \\ 2 College of Computer, Wuhan University, Wuhan 430072 , China \\ liss@whu.edu.cn \\ 3 The State Key Laborary of Software Engineering, Wuhan University, \\ Wuhan 430072, China
}

\begin{abstract}
Mobile agents are an important technology in e-commerce systems and offer new possibilities for the e-commerce applications. This paper examines some mobile agent integrity protocols and proposes a new protecting protocol of mobile agent integrity. It can defend most known attacks, provides encryption transmission and route secrecy of mobile agents.
\end{abstract}

\section{Introduction}

Mobile agents are an important technology in e-commerce systems and offer new possibilities for the e-commerce applications. They can provide very flexible approach for information gathering on prices and assets available from the hosts they visit. They can create new types of electronic ventures from e-shops, e-auctions to virtual enterprises and e-marketplaces. Such systems are developed for diverse business areas, e.g., contract negotiations, service brokering, stock trading and many others([1]) .

Mobile agent systems have many advantages over traditional distributed computing environments: require less network bandwidth, increase asynchrony among clients and servers, and dynamically update server interfaces, and introduce concurrency and so on ([2]). But certain applications have a need for protection of security of the mobile agents. In the mobile agent systems the agent's code and internal state autonomously migrate between hosts and could be easy changed during the transmission or at a malicious host site. A malicious host may expose, modify, insert, delete or truncate data the agent collected from other previously visited servers to benefit itself $([3,4])$.

The integrity of an agent means that its code and execution state can not be changed by an unauthorized party or such changes should be detectable. The general goal is to protect the results within the chain of partial results from being modified $([5,6,7])$. To protect integrity some protocols have been proposed in different

Please use the following format when citing this chapter:

Guan, H., Zhang, H., Chen, P., Zhou, Y., 2007, in IFIP International Federation for Information Processing, Volume 251, Integration and Innovation Orient to E-Society Volume1, Wang, W. (Lds), (Boston: Springer), pp. 194-201. 
papers. This paper will examine some protocols and extract general methods from these protocols. As result of this examination the paper will proposes a new integrity protocol for mobile agents. It can defend most known attacks, provides encryption transmission and route secrecy of mobile agents.

The rest of this paper is organized as follows. Section 2 describes related work. Section 3 describes the notations and security properties. Section 4 proposes a new integrity protocol for mobile agents. Section 5 gives security analysis of this protocol. Finally, conclusions are drawn in section 6.

\section{Related work}

Forward Integrity denotes the integrity of the partial results. Yee ([8]) defines the notion of weak forward integrity in the following mode "if a mobile agent visits a sequence of servers $S_{1}, S_{2}, \ldots, S_{n}$, and the first malicious server is $S_{m}$, then none of the partial results generated at servers $S_{i}$, where $i<m$, can be forged". In their scheme, an agent and its originator maintained a list of secret keys, or a key generating function. The agent used a key to encapsulate the collected offer and then destroyed the key. However, a malicious host may keep the key or the key generating function. When the agent revisits the host or visits another host conspiring with it, a previous offer or series of offers would be modified, without being detected by the originator.

Karjoth ([9]), et al. proposed a notion of strong forward integrity where an attacker $S_{m}$ can not forge any of partial results generated at server $S_{i}$, where $i<m$, even by colluding with one (or more) other visited server $S_{j}$, where $j<i$. In the their scheme, A chain $\mathrm{O}_{0}, \mathrm{O}_{1}, \mathrm{O}_{2}, \ldots, \mathrm{O}_{\mathrm{n}}$ is an ordered sequence of encapsulated offers such that each entry of the chain depends on the previous and the next members. This dependency is specified by a chaining relation. Their scheme could resist the modification attack but could not prevent two colluders truncation attack. In this attack, a host with the agent at hand colludes with a previously visited host to discard all entries between the two visits.

Cheng ([10]), et al. proposed a data collection protocol that prevents two colluders truncation attack in a free roaming agent. The protocol is to require an external party, typically the preceding visited host, to co-sign the agent migration. Therefore, two colluders are not sufficient to affect a truncation attack. Their scheme can also be generalized to prevent the $\mathrm{L}(\mathrm{L} \geq 2)$ colluder truncation attack. The cosigning mechanism But it could not prevent more than $\mathrm{L}$ colluders truncation attack.

Darren Xu ([11]), et al. proposed a scheme uses "one hop backwards and two hops forwards" chain relation as the protocol core to implement the generally accepted mobile agents security properties. This scheme can defend most known attacks. But if itinerary of mobile agents is protected, it difficult to find the second host forward. 


\section{Notations and security properties}

Table 2. The Notation Used in This Paper

\begin{tabular}{|c|c|}
\hline Notations & Meaning \\
\hline$\left(\mathrm{l}_{\mathrm{A}}, \mathrm{C}_{\mathrm{A}}, \mathrm{S}_{\mathrm{A}}, \mathrm{D}_{\mathrm{A}}\right)$ & $\begin{array}{l}\mathrm{I}_{A} \text { is } A^{\prime} \text { 's identity, } C_{A} \text { is } A^{\prime} \text { 's code, } S_{A} \text { is the state of } A \text { and } \\
D_{A} \text { is } A^{\prime} \text { 's data }\end{array}$ \\
\hline$S_{0}=S_{n+1}$ & ID of the originator \\
\hline $\mathrm{S}_{\mathrm{i}}, \mathrm{l} \leq \mathrm{i} \leq \mathrm{n}$ & ID of the host $i$ \\
\hline $\mathrm{T}$ & ID of the trusted third party \\
\hline $\mathrm{o}_{0}$ & $\begin{array}{l}\text { A secret possessed by host } S_{0} \text {. It can be regarded as a } \\
\text { dummy offer and is only known to the originator }\end{array}$ \\
\hline $\mathrm{o}_{\mathrm{i}}, \mathrm{l} \leq \mathrm{i} \leq \mathrm{n}$ & An offer from host $S_{i}$ \\
\hline $\mathrm{O}_{\mathrm{i}}, 0 \leq \mathrm{i} \leq \mathrm{n}$ & $\begin{array}{l}\text { An encapsulated offer (cryptographically protected } o_{i} \text { ) } \\
\text { from host } S_{i}\end{array}$ \\
\hline $\mathrm{O}_{0}, \mathrm{O}_{1}, \ldots, \mathrm{O}_{\mathrm{n}}$ & $\begin{array}{l}\text { The chain of encapsulated offers from the originator and } \\
\text { host } S_{1}, S_{2}, \ldots, S_{n}\end{array}$ \\
\hline $\mathrm{h}_{\mathrm{A}}$ & The agent integrity check value \\
\hline $\mathrm{h}_{\mathrm{i}}, 0 \leq \mathrm{i} \leq \mathrm{n}$ & Message integrity check value associated with $\mathrm{O}_{i}$ \\
\hline $\mathbf{r}_{\mathbf{i}}, 0 \leq \mathrm{i} \leq \mathbf{n}$ & A random number generated by host $S_{i}$ \\
\hline $\begin{array}{l}\left(\mathrm{KD}_{\mathrm{i}}, \mathrm{KE}_{\mathrm{i}}\right), 0 \leq \\
\mathrm{i} \leq \mathrm{n}\end{array}$ & A private/public key pair of host $S_{i}$ \\
\hline$\left(\mathrm{KD}_{\mathrm{T}}, \mathrm{KE}_{\mathrm{T}}\right)$ & A private/public key pair of $T$ \\
\hline $\operatorname{Enc}_{\mathrm{KE}_{1}}(\mathrm{~m})$ & $\begin{array}{l}\text { A message } m \text { asymmetrically encrypted with the public } \\
\text { key } K_{i} \text { of host } S_{i}\end{array}$ \\
\hline $\operatorname{Dec}_{\mathrm{KD}_{1}}(\mathrm{~m})$ & $\begin{array}{l}\text { A message } m \text { asymmetrically decrypted with the private } \\
\text { key } K D_{i} \text { of } T\end{array}$ \\
\hline $\operatorname{Sig}_{\mathrm{KD}_{\mathrm{j}}}(\mathrm{m})$ & $\begin{array}{l}\text { The signature of host } \mathrm{Si} \text { on a message } m \text { using its private } \\
\text { key } \mathrm{KD}_{\mathrm{i}} \text {. }\end{array}$ \\
\hline Verif $\left(\sigma, \mathrm{KE}_{\mathrm{i}}\right)$ & $\begin{array}{l}\text { A signature verification function for signature } \sigma \text { and public } \\
\text { key } \mathrm{KE}_{\mathrm{i}}\end{array}$ \\
\hline$H(m)$ & A one-way collision-resistant hash function \\
\hline $\mathrm{A} \rightarrow \mathrm{B}: \mathrm{m}$ & A sending a message $m$ to $B$ \\
\hline
\end{tabular}

An agent is defined as $A=\left(I_{A}, C_{A}, S_{A}, D_{A}\right)$ where $I_{A}$ is the identity, $C_{A}$ is the code, $S_{A}$ is the state and $D_{A}$ is the data of the agent. Both $I_{A}$ and $C_{A}$ are static while $\mathrm{S}_{\mathrm{A}}$ and $\mathrm{D}_{\mathrm{A}}$ are variable.

Digital signature and encryption need a working public key infrastructure. Each host $\mathrm{S}_{\mathrm{i}}$ has a certified private/public key pair $\left(\mathrm{KD}_{\mathrm{i}}, \mathrm{KE}_{\mathrm{i}}\right)$. The transmission of mobile agents is encrypted. An agent's route information is secret. The main technique is to require a trusted third party. 
Assume that an agent has visited an undetermined number $m$ of hosts, $\mathrm{m} \leq \mathrm{n}$. An agent is captured by an attacker. This attacker possibly is the host $\mathrm{S}_{\mathrm{m}+1}$. Some hosts excluding $S_{m}$ may collude with the attacker. Let i range over $1, \ldots, m$. Mobile agents security properties based on the assumptions:

- Verifiable Forward Integrity: The trust third party $T$ can verify the offer $o_{i}$ by checking whether the chain is valid at $\mathrm{O}_{\mathrm{i}}$.

- Data Confidentiality: Only the originator can extract the offers $o_{i}$ from the encapsulated offers $\mathrm{O}_{i}$.

- Non-repudiability: Host $S_{\mathbf{i}}$ cannot deny submitting $o_{\mathbf{i}}$ once it has been received by originator $S_{0}$.

- Forward Privacy: None of the identities of the creator of offer $o_{i}$ can be extracted.

- Strong Forward Integrity: None of the encapsulated offers $O_{k}$, where $k \leq m$, can be modified.

- Insertion Resilience: No offer can be inserted at i unless explicitly allowed, i.e., $S_{m+1}$. It is not possible for $S_{m+1}$ to insert more than one offer even if $S_{m+1}$ collude with some specific $L$ hosts.

- Deletion Resilience: No partial result $O_{k}$ can be deleted by any $S_{i}$, with $k<$ $\mathrm{m}$. It is not possible for $\mathrm{S}_{\mathrm{m}+1}$ to delete more than one offer even if $S_{m+1}$ collude with some specific $L$ hosts.

- Truncation Resilience: Truncation at $i$ is not possible.

- Itinerary Secrecy: Only the originator and the trusted third party T know a mobile agent's migration route. Truncation at $i$ is not possible even if some specific L hosts collude with $S_{i}$ to carry out the attack.

- Secure Transmission.

\section{The Protocol}

\subsection{Agent at the originator $S_{0}$ :}

$$
\begin{aligned}
\mathrm{S}_{0}: \quad & \mathrm{O}_{0}=\operatorname{Sig}_{\mathrm{KD}_{0}}\left(\operatorname{Enc}_{\mathrm{KE}_{0}}\left(\mathrm{o}_{0}, \mathrm{r}_{0}\right)\right) \\
& \mathrm{h}_{0}=\operatorname{Sig}_{\mathrm{KD}_{0}}\left(\mathrm{H}\left(\mathrm{O}_{0}\right), \operatorname{Enc}_{\mathrm{KE}_{\mathrm{T}}}\left(\mathrm{S}_{\mathrm{l}}\right)\right) \\
& \mathrm{h}_{\mathrm{A}}=\operatorname{Sig}_{\mathrm{KD}_{0}}\left(\mathrm{H}\left(\mathrm{I}_{\mathrm{A}} \| \mathrm{C}_{\mathrm{A}}\right)\right) \\
\mathrm{S}_{0} \rightarrow \mathrm{T}: & \mathrm{h}_{0} \\
\mathrm{~S}_{0} \rightarrow & \mathrm{S}_{1}: \operatorname{Enc}_{\mathrm{KE}_{\mathrm{1}}}\left(\mathrm{I}_{\mathrm{A}}\left\|\mathrm{C}_{\mathrm{A}}\right\| \mathrm{S}_{\mathrm{A}} \| \mathrm{D}_{\mathrm{A}}\right), \mathrm{h}_{\mathrm{A}}, \mathrm{O}_{0}
\end{aligned}
$$

\subsection{Agent at host $S_{1}$ :}

$$
\begin{aligned}
& \mathrm{S}_{1}: \quad \operatorname{Dec}_{\mathrm{KE}_{1}}\left(\mathrm{I}_{\mathrm{A}}\left\|\mathrm{C}_{\mathrm{A}}\right\| \mathrm{S}_{\mathrm{A}} \| \mathrm{D}_{\mathrm{A}}\right) \\
& \operatorname{Verif}\left(\mathrm{h}_{\mathrm{A}}, \mathrm{KE}_{0}\right) \stackrel{?}{=} \text { true }
\end{aligned}
$$




$$
\begin{aligned}
\mathrm{O}_{\mathrm{t}}=\operatorname{Sig}_{\mathrm{KD}_{1}}\left(\operatorname{Enc}_{\mathrm{KE}_{0}}\left(\mathrm{O}_{1}, \mathrm{r}_{1}\right)\right) \\
\mathrm{h}_{1}=\operatorname{Sig}_{\mathrm{KD}_{1}}\left(\mathrm{H}\left(\mathrm{O}_{1}\right), \operatorname{Enc}_{\mathrm{KE}_{\mathrm{T}}}\left(\mathrm{S}_{2}\right)\right) \\
\mathrm{S}_{1} \rightarrow \mathrm{T}: \mathrm{h}_{1} \\
\mathrm{~S}_{1} \rightarrow \mathrm{S}_{2}: \operatorname{Enc}_{\mathrm{KE}_{2}}\left(\mathrm{I}_{\mathrm{A}}\left\|\mathrm{C}_{\mathrm{A}}\right\| \mathrm{S}_{\mathrm{A}} \| \mathrm{D}_{\mathrm{A}}\right), \mathrm{h}_{\mathrm{A}},\left\{\mathrm{O}_{0}, \mathrm{O}_{1}\right\}
\end{aligned}
$$

\subsection{Agent at host $S_{\mathrm{i}}$ :}

$$
\begin{aligned}
& \mathrm{S}_{\mathrm{i}}: \quad \operatorname{Dec}_{\mathrm{KE}_{\mathrm{i}}}\left(\mathrm{I}_{\mathrm{A}}\left\|\mathrm{C}_{\mathrm{A}}\right\| \mathrm{S}_{\mathrm{A}} \| \mathrm{D}_{\mathrm{A}}\right) \\
& \operatorname{Ver}\left(\mathrm{h}_{\mathrm{A}}, \mathrm{KE}_{0}\right) \stackrel{?}{=} \text { true } \\
& \mathrm{O}_{\mathrm{i}}=\operatorname{Sig}_{\mathrm{KD}_{i}}\left(\operatorname{Enc}_{\mathrm{KE}_{0}}\left(\mathrm{o}_{i}, \mathrm{r}_{\mathrm{i}}\right)\right) \\
& \mathrm{h}_{\mathrm{i}}=\mathrm{Sig}_{\mathrm{KD}_{\mathrm{i}}}\left(\mathrm{H}\left(\mathrm{O}_{\mathrm{i}}\right), \operatorname{Enc}_{\mathrm{KE}}\left(\mathrm{S}_{\mathrm{i}+1}\right)\right) \\
& \mathrm{S}_{\mathrm{i}} \rightarrow \mathrm{T}: \mathrm{h}_{\mathrm{i}} \\
& \mathrm{S}_{\mathrm{i}} \rightarrow \mathrm{S}_{\mathrm{i}+1}: \operatorname{Enc}_{\mathrm{KE}_{\mathrm{i}+1}}\left(\mathrm{I}_{\mathrm{A}}\left\|\mathrm{C}_{\mathrm{A}}\right\| \mathrm{S}_{\mathrm{A}} \| \mathrm{D}_{\mathrm{A}}\right), \mathrm{h}_{\mathrm{A}},\left\{\mathrm{O}_{\mathrm{k}} \mid 0 \leq \mathrm{k} \leq \mathrm{i}\right\}
\end{aligned}
$$

\subsection{Agent at host $S_{n}$ :}

$$
\begin{aligned}
\mathrm{S}_{\mathrm{n}}: \quad & \operatorname{Dec}_{\mathrm{KE}_{\mathrm{n}}}\left(\mathrm{I}_{\mathrm{A}}\left\|\mathrm{C}_{\mathrm{A}}\right\| \mathrm{S}_{\mathrm{A}} \| \mathrm{D}_{\mathrm{A}}\right) \\
& \operatorname{Ver}\left(\mathrm{h}_{\mathrm{A}}, \mathrm{KE}_{0}\right) \stackrel{?}{=} \text { true } \\
& \mathrm{O}_{\mathrm{n}}=\operatorname{Sig}_{\mathrm{KD}_{\mathrm{n}}}\left(\operatorname{Enc}_{\mathrm{KE}_{\mathrm{g}}}\left(\mathrm{O}_{\mathrm{n}}, \mathrm{r}_{\mathrm{n}}\right)\right) \\
& \mathrm{h}_{\mathrm{n}}=\operatorname{Sig}_{\mathrm{KD}_{\mathrm{n}}}\left(\mathrm{H}\left(\mathrm{O}_{\mathrm{n}}\right), \operatorname{Enc}_{\mathrm{KE}_{\mathrm{T}}}\left(\mathrm{S}_{\mathrm{n}+1}\right)\right) \\
\mathrm{S}_{\mathrm{n}} \rightarrow \mathrm{T}: \mathrm{h}_{\mathrm{n}} & \\
\mathrm{S}_{\mathrm{n}} \rightarrow & \mathrm{S}_{\mathrm{n}+1}: \operatorname{Enc}_{\mathrm{KE}_{\mathrm{n} \cdot 1}}\left(\mathrm{I}_{\mathrm{A}}\left\|\mathrm{C}_{\mathrm{A}}\right\| \mathrm{S}_{\mathrm{A}} \| \mathrm{D}_{\mathrm{A}}\right), \mathrm{h}_{\mathrm{A}},\left\{\mathrm{O}_{k} \mid 0 \leq \mathrm{k} \leq \mathrm{n}\right\}
\end{aligned}
$$

\subsection{Agent at host $S_{n+1}\left(S_{n+1}=S_{0}\right)$ :}

$$
\begin{aligned}
\mathrm{S}_{\mathrm{n}+1}: & \operatorname{Dec}_{\mathrm{KE}_{\mathrm{n}+1}}\left(\mathrm{I}_{\mathrm{A}}\left\|\mathrm{C}_{\mathrm{A}}\right\| \mathrm{S}_{\mathrm{A}} \| \mathrm{D}_{\mathrm{A}}\right) \\
& \operatorname{Ver}\left(\mathrm{h}_{\mathrm{A}}, \mathrm{KE}_{0}\right) \stackrel{?}{=} \text { true }
\end{aligned}
$$

$\mathrm{S}_{\mathrm{n}+1} \rightarrow \mathrm{T}:\left\{\mathrm{h}_{\mathrm{k}}^{\prime}=\mathrm{H}\left(\mathrm{O}_{\mathrm{k}}\right) \mid 0 \leq \mathrm{k} \leq \mathrm{n}\right\}, \mathrm{T}$ verifies the forward integrity and returns results to host $S_{n+1}$

\subsection{At the trusted third party $T$ :}

$\mathrm{T}: \quad$ Verif $\left(\mathrm{h}_{\mathrm{i}}, \mathrm{KE}_{\mathrm{i}}\right)$, recover $\mathrm{H}\left(\mathrm{O}_{\mathrm{i}}\right), \mathrm{Enc}_{\mathrm{KE}_{\mathrm{T}}}\left(\mathrm{S}_{\mathrm{i}+1}\right)$ 


$$
\begin{aligned}
& \operatorname{Dec}_{\mathrm{Kd}_{\mathrm{r}}}\left(S_{i+1}\right), \text { recover } S_{i+1} \\
& \text { Receive } h_{0}^{\prime}, h_{1}^{\prime}, h_{2}^{\prime}, \ldots, h_{n}^{\prime} \\
& h_{k}^{\prime} \stackrel{?}{=} H\left(O_{k}\right),(0 \leq k \leq n)
\end{aligned}
$$

To begin the protocol, the originator $S_{0}$ randomly generates $r_{0}$. Host $S_{0}$ encrypts a dummy offer $\mathrm{o}_{0}$ and $\mathrm{r}_{0}$ using its own public key $\mathrm{KE}_{0}$. Host $\mathrm{S}_{0}$ signs this encrypted value to construct a dummy encapsulated offer $\mathrm{O}_{0}$. Next, Host $\mathrm{S}_{0}$ calculates a hash value $h_{0}$ from $O_{0}$, and encrypts $S_{1}$ using T's public key $K E_{T}$, and then signs them. Host $S_{0}$ also computes a hashed value $h_{A}$ from $I_{A}$ and $C_{A} \cdot h_{A}$ is the certified agent integrity checksum. Host $S_{0}$ encrypts this agent using it's the next host's public key $\mathrm{KE}_{3}$. Finally, Host $\mathrm{S}_{0}$ sends $h_{0}$ to the trusted third party $T$ and the agent migrates to the first host $S_{1}$.

When the agent arrives at host $S_{i}, S_{i}$ verifies $h_{A}$ in order to ensure the identity $I_{A}$ and code $C_{A}$ were not modified by any malicious hosts. Host $S_{i}$ randomly generates $r_{i}$. Host $S_{i}$ encrypts $o_{i}$ and $r_{i}$ using the originator's public key $K_{0}$. Host $S_{i}$ signs this encrypted value to construct an encapsulated offer $\mathrm{O}_{i}$. Host $\mathrm{S}_{i}$ calculates a hash value $h_{i}$ from $O_{i}$, and encrypts $S_{i+1}$ using T's public key $\mathrm{KE}_{\mathrm{T}}$, and then signs them. Finally, Host $S_{i}$ sends $h_{i}$ to the trusted third party $T$ and the agent migrates to host $S_{i+1}$.

When the agent returns host $S_{n+1}\left(S_{n+1}=S_{0}\right), S_{n+1}$ verifies $h_{A}$ again. Host $S_{n+1}$ computes a hash value $h_{k}^{\prime}$ from $O_{k}(0 \leq k \leq n)$, then sends $h_{k}^{\prime}$ to the trusted third party $T$ and requests $T$ to verify the forward integrity.

The trusted third party $T$ receives $h_{i}$, recovers $H\left(O_{i}\right)$ and $S_{i+1}$. The chain of hash $\mathrm{H}\left(\mathrm{O}_{0}\right), \mathrm{H}\left(\mathrm{O}_{1}\right), \mathrm{H}\left(\mathrm{O}_{2}\right), \ldots, \mathrm{H}\left(\mathrm{O}_{\mathrm{n}}\right)$ is an ordered sequence. $\mathrm{S}_{1}, \mathrm{~S}_{2}, \ldots, \mathrm{S}_{\mathrm{n}}$ is the agent's route information.

$T$ receives $h_{k}^{\prime}(0 \leq k \leq i-1)$. It compares $h_{k}^{\prime}$ with $H\left(O_{k}\right)$, so as to ensure $O_{k}$ was not altered. Then $T$ returns results to host $S_{n+l}$.

\section{Security Analysis}

Here we analyze how the protocol achieves the security properties.

- Verifiable Forward Integrity: The trust third party $T$ fulfills the forward integrity for each host $S_{i}$.

- Data Confidentiality: If the encryption scheme is secure, only the originator $\mathrm{S}_{0}$ can decrypt $\operatorname{Enc}_{\mathrm{KE}_{0}}\left(\mathrm{o}_{\mathrm{i}}, \mathrm{r}_{\mathrm{i}}\right)$ to extract $\mathrm{o}_{i}$. The trusted third party $\mathrm{T}$ and other hosts cannot gain $\mathrm{O}_{i}$.

- Non-repudiability: Each host $S_{i}$ signs its offer $o_{i}$ by its private key $K_{D_{i}}$. If the signature scheme is secure, host $S_{i}$ cannot repudiate $O_{i}$.

- Forward Privacy: The host identity $S_{i}$ is encrypted using the trust third party $T$ 's public key. Only $T$ can extract the identity of $S_{1} . T$ saves the agent migrate route.

- Strong Forward Integrity: Suppose the attacker leaves $O_{m}$ intact but changes $O_{k}$ to $O_{k}^{\prime}$, where $0 \leq k \leq m-1$. $S_{n+1}$ will calculate $h_{k}^{\prime}$ from $O_{k}^{\prime}$ and send $h_{k}^{\prime}$ 
to $T$. In the trusted third party $T$, Since $h_{k}^{\prime}$ not equal $H\left(O_{k}\right)$, T will report this attack to $\mathrm{S}_{\mathrm{m}+\mathrm{l}}$.

- Insertion Resilience: Suppose the attacker leaves $\mathrm{O}_{m}$ intact but inserts a $\mathrm{O}_{k}^{\prime}$ before $O_{k}$, where $0 \leq \mathrm{k} \leq \mathrm{m}-1$. Following similar reasoning as in the above analysis, $S_{n+1}$ will calculate $h_{k}^{\prime}$ from $O_{k}^{\prime}$ and send $h_{k}^{\prime}$ to the trust third party $T$. Through comparing $h_{k}^{\prime}$ with $H\left(O_{k}\right)$, T will find this change. Therefore no offer can be inserted in the chain of encapsulated offers.

- Deletion Resilience: Suppose the attacker leaves $O_{m}$ intact but deletes $O_{k}$, where $0 \leq k \leq m-1$. $S_{n+1}$ will calculate $h_{k}$ from $O_{k+1}$ and send $h_{k}$ to the trust third party $T$. Through comparing $h_{k}^{\prime}$ with $H\left(O_{k}\right)$, T will find this change.

- Truncation Resilience: Suppose the attacker leaves $\mathrm{O}_{m}$ intact but deletes $\mathrm{O}_{k}$, where $0 \leq k \leq m-1$. $S_{n+1}$ will calculate $h_{k}^{\prime}$ from $O_{k+1}$. Similarly, T will find this modify. In the other words, if the $T$ is secure, Collusion attack is infructuous.

- Itinerary Secrecy: Only the originator and the trust third party $\mathrm{T}$ know a mobile agent's migration route.

- Secure Transmission: The transmission of mobile agents is encrypted.

\section{Conclusions}

This paper examined some protocols and gives security requirements in mobile agent systems. As result of this examination the paper will proposes a new integrity protocol for mobile agents. It can defend most known attacks, provides encryption transmission and route secrecy of mobile agents.

\section{Acknowledgement}

This research is Supported by the National Natural Science Foundation of China (60673071)

\section{References}

1. A. Corradi, M. Cremonini, R. Montanari and C. Stefanelli, Mobile Agents Integrity for Electronic Commerce Applications, Information Systems Vol. 24, No. 6, pp. 519-533 (1999).

2. D.B. Lange and M. Oshima, Seven Good Reasons for Mobile Agents, Communications of the ACM, 1999, 42(3): 88-89.

3. W. Jansen, Countermeasures for Mobile Agent Security, Computer Communications, 2000, 23(17): 1667-1676.

4. S. Fünfrocken, Protecting Mobile Web-Commerce Agents with Smartcards, First International Symposium on Agent Systems and Applications (ASA'99)/Third International Symposium on Mobile Agents (MA'99), Palm Springs (1999).

5. D. Westhoff, et al, Protecting a Mobile Agent's Route against Collusions, Proceedings of SAC'99, San Antonio: Springer-Verlag, 1999: 215-226.

6. M. Giansiracusa, Mobile Agent Protection Mechanisms and the Trusted Agent Proxy Server (TAPS) Architecture,

(2003). http://www isi.qut.edu.au/research/publications/technical/qut-isrc-tr-2003-010.pdf. 
7. P. J. Hardjono and J. Seberry, Fundamentals of Computer Security, Springer-Verlag, Berlin (2003).

8. B.S. Yee, A Sanctuary for Mobile Agents, Proceedings of Secure Internet Programming: Security Issues for Distributed and Mobile Objects, Berlin: Springer-Verlag, 1999: 261273.

9. G. Karjoth, N. Asokan, and C. Gülcü, Protecting the Computation Results of Freeroaming Agents, Proceedings of the 2nd International Workshop on Mobile Agents (MA '98), Stuttgart: Springer-Verlag, 1998: 195-207.

10. J.S.L. Cheng and K.W. Victor, Defenses against the Truncation of Computation Results of Free-roaming Agents, Proceedings of the 4th International Conference on Information and Communications Security, Singapore: Springer-Verlag, 2002: 1-12.

11. X. Darren, An improved free-roaming mobile agent security protocol against colluded trunation attack, Proceedings of the 30th Annual International Computer Software and Applications Conference (2006). 